\title{
Spontaneous tumor lysis syndrome in diffuse large B-cell lymphoma patient as a cause of acute kidney injury
}

\section{Przemysław Kwiatkowski' , Grzegorz Kade², Janusz Hałka'}

${ }^{1}$ Department of Hematology, Warmian-Masurian Cancer Center of the Ministry of the Interior and Administration's Hospital in Olsztyn, Poland

${ }^{2}$ Warmian-Masurian Cancer Center of the Ministry of the Interior and Administration's Hospital in Olsztyn, Poland

\section{Correspondence:}

Grzegorz Kade

Warmian-Masurian Cancer Center of the Ministry of the Interior and Administration's Hospital in Olsztyn, Poland 10-228 Olsztyn, Wojska Polskiego 37 phone: 895398000

fax: 895398240

e-mail:grzegorz.kade@poliklinika.net

Received:

31.03.2021

Accepted:

14.04.2021

DOI: 10.24292/01.OR.121140421

Copyright $\odot$ Medical Education.

All rights reserved.
ABSTRACT

Idiopathic tumor lysis syndrome is a rare complication in the course of neoplastic disease. This condition requires an interdisciplinary therapeutic procedure. The presented case of spontaneous tumor lysis syndrome in the course of malignant large B-cell lymphoma describes an effective therapeutic approach in this type of cases.

Key words: spontaneous tumor lysis syndrome, interdisciplinary therapeutic procedure, acute kidney injury 


\section{INTRODUCTION}

One of the most common causes of acute kidney injury in hematology department is the tumor lysis syndrome (TLS) [1, 2]. The syndrome is a life-threatening metabolic disorder resulting from the rapid breakdown of cancer cells. The first description of the TLS dates back to 1929 and concerned the patient diagnosed with chronic lymphocytic leukemia [3]. The classification and definition of TLS is based on the Cairo-Bishop criteria, which distinguish between laboratory TLS and clinical TLS [4]. Spontaneous tumor lysis syndrome (STLS) is a rare event and was first described in 1977 in the course of disseminated gastric cancer [5]. STLS can be diagnosed when TLS is manifested in the absence of active chemotherapy. This case describes a diffuse large B-cell lymphoma patient who presented a pathological mass in the craniofacial region and was diagnosed with STLS.

\section{CASE PRESENTATION}

A 60-year-old Caucasian male with a history of hypertension and diabetes mellitus, was admitted to the hematology ward due to pathological mass of the craniofacial region which was histopathologically diagnosed as diffuse large B cell lymphoma (IHC: CD20+, bcl2+, bcl6+, $\mathrm{Ki}-67$ positive in $95 \%$ of cells), in order to perform staging and initiate treatment. The patient complained of a growing mass of the right side of the craniofacial region that initially appeared as a small bulge of the gingiva of the right side of maxilla approximately 2 months before admission and started to grow extensively in the last month (fig. 1). In addition the patient noticed few kilograms loss of body weight and sporadically night sweats. The patient had no history of decreased urine output prior to admission. On admission his vital signs were a pulse of 82 beats per minute, respiratory rate of 16 breaths per minute, blood pressure of 102/55 millimeters of mercury, temperature of $36,7^{\circ} \mathrm{C}$ and oxygen saturation of $97 \%$ on room air. Physical examination revealed a large pathological mass in the right craniofacial region and the oral cavity as well as obesity (BMI $37,2 \mathrm{~kg} / \mathrm{m}^{2}$ ). Laboratory examination on admission showed high levels of creatinine, uric acid, C-reactive protein as well as dyselectrolytemia. Interestingly, the level of lactate dehydrogenase did not exceed the reference range and was $140 \mathrm{U} / \mathrm{I}$. Based on clinical and laboratory criteria the patient was diagnosed with STLS.

The PET-CT scan showed a large tumor of the size $77 \times 55 \times$ $60 \mathrm{~mm}$ on the right side of the craniofacial region (fig. 2A, B). The pathological mass caused bone destruction, filled the space of the maxillary sinus, penetrated the cheek region and extended into the palate. The CT scans of neck, thorax, abdomen and pelvis
FIGURE 1.

Image showing tumor mass on the right side of the craniofacial region prior to initiating chemotherapy.
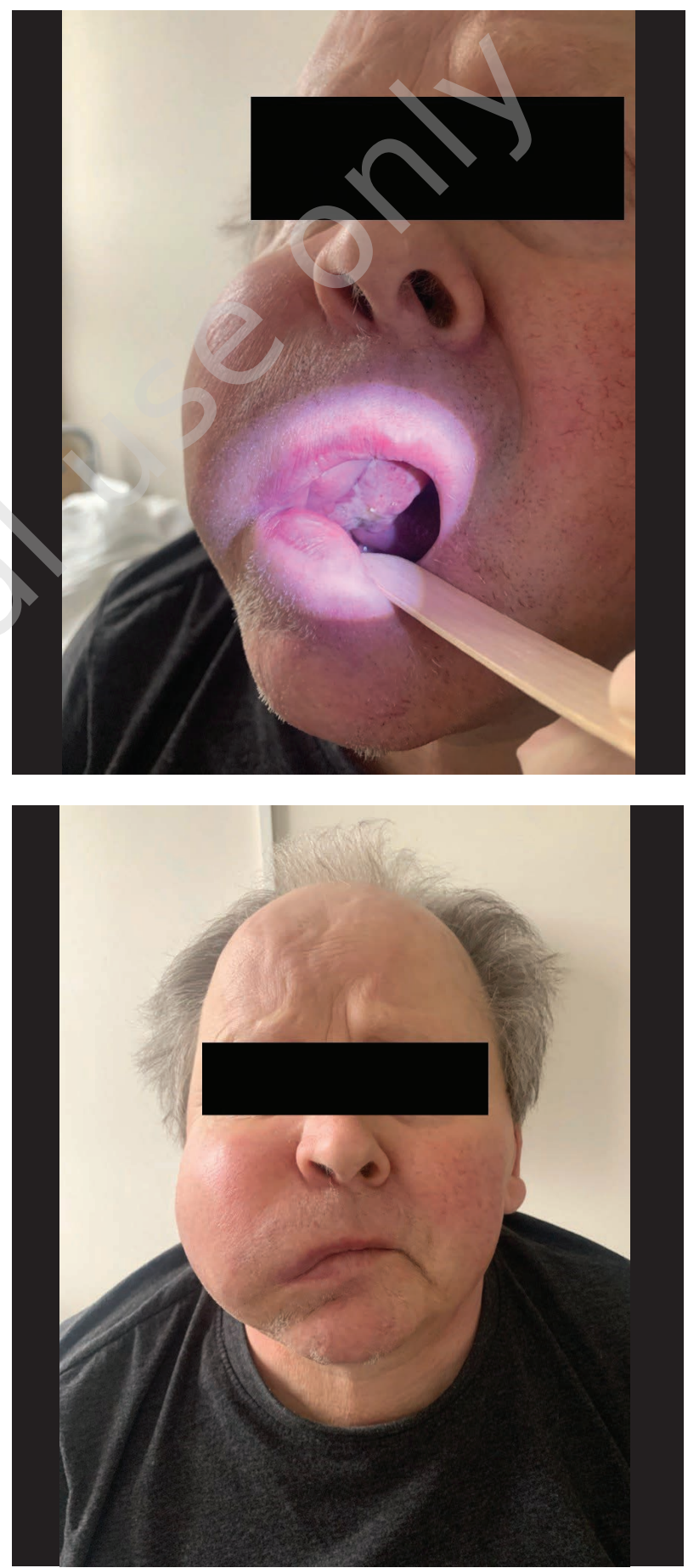

revealed no pathologies. Transthoracic echocardiogram showed slight distention of both atria, normal left ventricular size with estimated ejection fraction of $65 \%$. Flow cytometry of bone marrow and cerebrospinal fluid revealed no infiltration with lymphoma cells. 
FIGURE 2.

$2 \mathrm{~A}(\mathrm{CT})$ and $2 \mathrm{~B}$ (FDG-PET) showing tumor mass on the right side of the craniofacial region prior to initiating chemotherapy.
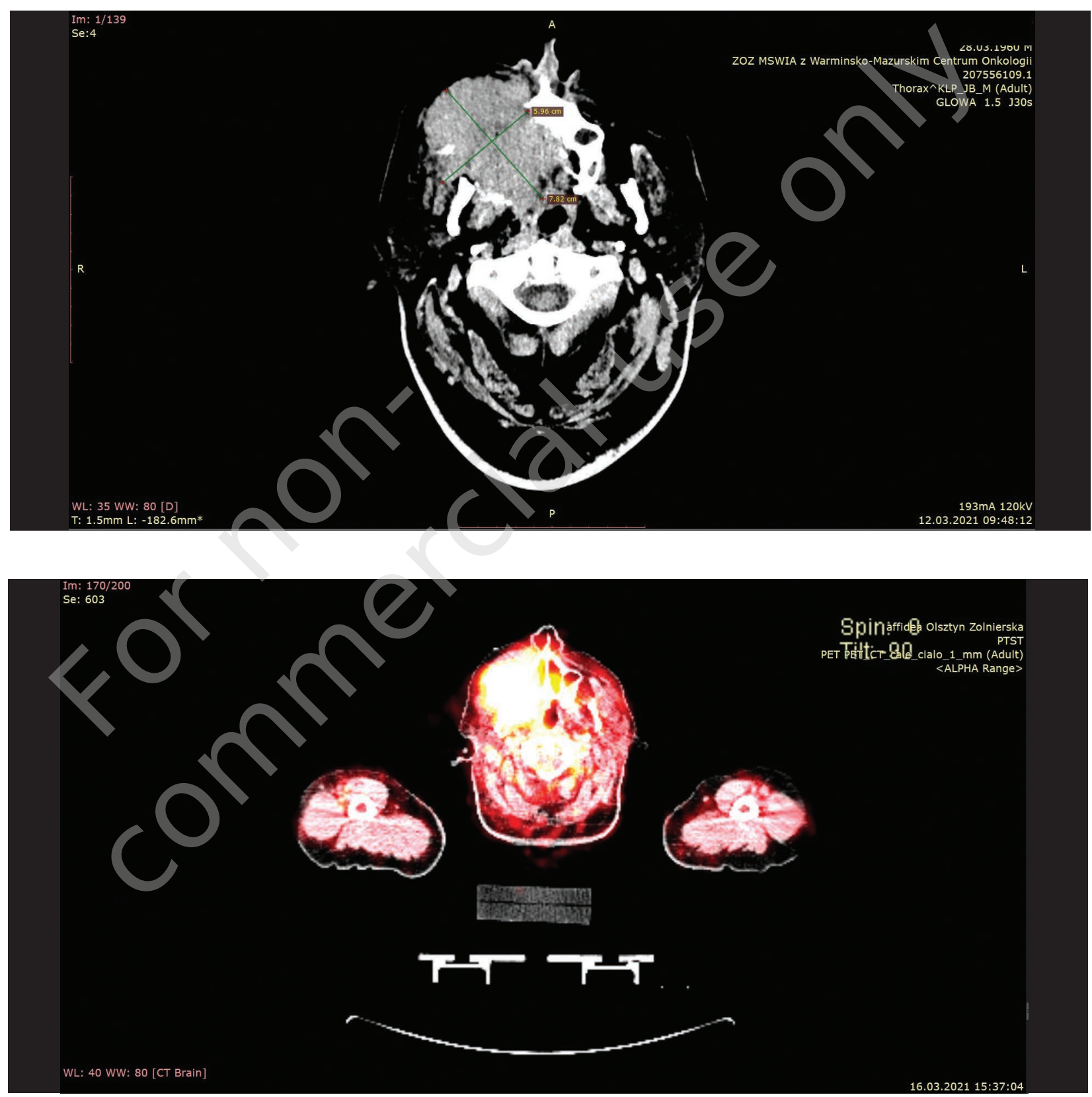

The patient's complete blood count and comprehensive metabolic panel trend throughout hospitalization period are summarized in the table 1.

The trends of serum phosphorus, serum creatinine, and serum uric acid are shown respectively in the figure 3.
On the day of admission the patient started intravenous hydration and was given rasburicase. Pretreatment phase with $20 \mathrm{mg}$ intravenous dexamethasone (days 1-7) was initiated. In the following days the patient's kidney function improved as well a significant drop in uric acid levels was observed thus an emergent hemodialysis was necessary. The patient started intravenous 
TABLE 1.

Complete blood count and comprehensive metabolic panel trend since hospitalization onset.

\begin{tabular}{|c|c|c|c|c|c|c|c|c|c|c|c|}
\hline Parameter & Day 1 & Day 2 & Day 3 & Day 4 & Day 5 & Day 6 & Day 7 & Day 8 & Day 9 & Day 10 & Day 11 \\
\hline WBC & 10.96 & & & 7.62 & & 8.25 & & 7.05 & 6.66 & 3.81 & 4.14 \\
\hline RBC & 4.54 & & & 4.37 & & 4.42 & & 4.32 & 4.16 & 4.23 & 4.34 \\
\hline HGB & 13.0 & & & 12.3 & & 12.7 & & 12.5 & 11.9 & 12.0 & 12.4 \\
\hline HCT & 38.3 & & & 36.6 & & 36.9 & & 36.8 & 35.2 & 36.1 & 36.5 \\
\hline Platelets & 360 & & & 359 & & 376 & & 354 & 335 & 279 & 254 \\
\hline Uric acid & 10.0 & 1.4 & & & 2.7 & & & & 3.0 & & \\
\hline Bilirubin & 1.09 & & & & & & & & 1.29 & 1.36 & 1.04 \\
\hline C-reactive protein & 88 & & & & & 10 & & & & & \\
\hline Creatinine & 4.11 & 5.18 & 3.56 & 2.00 & 1.48 & 1.42 & 1.42 & 1.34 & 1.34 & 1.35 & 1.21 \\
\hline Sodium & 128 & 132 & 132 & 136 & 137 & 140 & 143 & 137 & 138 & 144 & 143 \\
\hline Potassium & 4.0 & 4.5 & 3.7 & 4.1 & 3.8 & 3.5 & 3.9 & 3.7 & 3.6 & 3.6 & 3.5 \\
\hline Chloride & 84 & 90 & 88 & 92 & 94 & 96 & 99 & 97 & 97 & 102 & 101 \\
\hline Calcium & 5.16 & 4.55 & 4.36 & 4.59 & 4.52 & 4.56 & 4.64 & 4.39 & 4.46 & 4.18 & 4.10 \\
\hline Phosphorus & 5.42 & 7.62 & 4.46 & 4.27 & 3.49 & 3.27 & 3.65 & 3.34 & 3.09 & 2.52 & 3.16 \\
\hline
\end{tabular}

FIGURE 3.

The trends of serum phosphorus, serum creatinine, and serum uric acid.

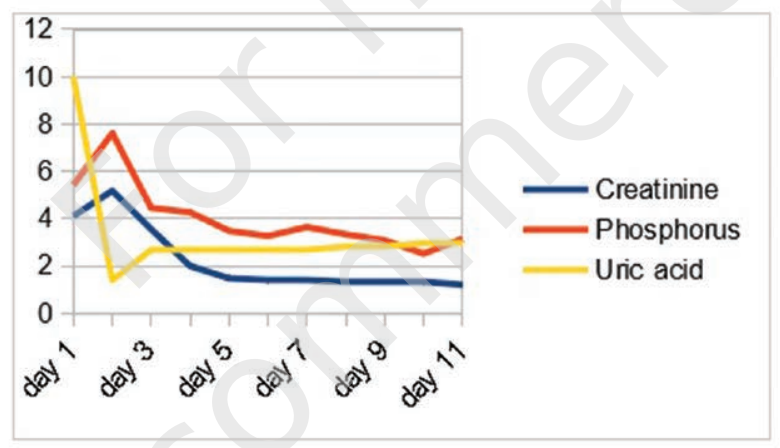

cyclophosphamide $200 \mathrm{mg}$ (days 6 to 8 after admission) and on day 9 of hospitalization the patient started on chemotherapy with rituximab $375 \mathrm{mg} / \mathrm{m}^{2}$. Cyclophosphamide $1000 \mathrm{mg}$, vincristine $2 \mathrm{mg}$, doxorubicin $100 \mathrm{mg}$ were given on the consecutive day. Methotrexate with dexamethasone intrathecally as a prophylaxis of central nervous system involvement as well as granulocyte colony-stimulating factor as prophylaxis for febrile neutropenia were administered. In anticipation for the second course of R-CHOP chemotherapy, the patient was discharged from hospital in good clinical condition on day 12 of hospitalization.

\section{DISCUSSION}

TLS most often occurs in the first days of chemotherapy, less often radiotherapy and concerns the neoplasms with high proliferative activity, especially hematological malignancies. This complication very rarely occurs spontaneously in the natural course of neoplastic disease before the initiation of chemotherapy, however SPTLS was observed more frequently in hematological malignancies including Burkitt's lymphoma [6], acute myeloid leukemia [7], acute lymphoblastic leukemia [8], anaplastic large T cell lymphoma [9] and myelofibrosis [10]. To date there have been only few reports of STLS in patients diagnosed with diffuse large B cell lymphoma. Our patient had a classic picture of STLS. Although at admission the laboratory investigation revealed that the patient suffered from renal failure, hyperuricemia, hyperkalemia, hypocalcemia and hyperphosphatemia, there was no history of decreased urine output. The patient started intravenous hydration and was administered rasburicase immediately after admission, what resulted in the rapid improvement of renal function together with metabolic parameters.

The treatment of TLS should include correction of fluid and electrolyte imbalance by forcing appropriate diuresis (hydration, loop diuretics), reduction of hyperphosphatemia (aluminum hydroxide, sevelamer hydrochloride), hyperkalemia (ion-exchange resins, insulin-glucose infusions, calcium gluconate), hypocalcemia (calcium gluconate) and hyperuricemia (allopurinol, rasburicase) [11]. Absolute indications for dialysis in patients with the diagnosis of TLS include: severe oliguria or anuria, therapy-resistant hyperkalemia and hyperphosphatemia, as well as symptomatic hyperphosphatemia-induced hypocalcemia [12]. The safety, effectiveness and tolerability of rasburicase in the prevention and treatment of TLS was confirmed in several studies. Rasburicase reduces rapidly uric acid levels both in pediatric and adult patients and lowers the risk of TLS development. Several studies demonstrated the efficacy of rasburicase to normalize or decrease creatinine levels thus to reduce the necessity of dialysis $[13,14]$ as in the presented case. 


\section{CONCLUSION}

In conclusion, STLS should be suspected in all patients with hyperuricemic acute renal failure. STLS is reversible when recognized early and treated appropriately as in the present patient.

\section{References}

1. Darmon $M$, Vincent $F$, Camous $L$ et al. Tumour lysis syndrome and acute kidney injury in high-risk haematology patients in the rasburicase era. A prospective multicentre study from the Groupe de Recherche en Réanimation Respiratoire et Onco-Hématologique. Br J Haematol. 2013; 162(4): 489-97.

2. Lahoti A, Kantarjian H, Salahudeen AK et al. Predictors and outcome of acute kidney injury in patients with acute myelogenous leukemia or high-risk myelodysplastic syndrome. Cancer. 2010; 116(17): 4063-8.

3. Bedrna J, Polcák J. Actuary ureter closure after irradiation with x-rays for chronic leukemias. Medizinische Klinik. 1929; 25: 1700-1.

4. Cairo MS, Bishop M. Tumour lysis syndrome: new therapeutic strategies and classification. Br J Haematol. 2004; 127(1): 3-11.

5. Crittenden DR, Ackerman GL. Hyperuricemic Acute Renal Failure in Disseminated Carcinoma. Arch Intern Med. 1977; 137(1): 97-9. http://doi. org/10.1001/archinte.1977.03630130065016.

6. Jasek AM, Day HJ. Acute spontaneous tumor lysis syndrome. Am J Hematol. 1994; 47: 129-31.

7. Lotfi M, Brandwein JM. Spontaneous acute tumor lysis syndrome in acute myeloid leukemia? A single case report with discussion of the literature. Leuk Lymphoma. 1998; 29: 625-8.

8. Kobayashi D, Wofford MM, McLean TW et al. Spontaneous tumor lysis syndrome in a child with T-cell acute lymphoblastic leukemia. Pediatr Blood Cancer. 2010; 54(5): 773-5. http://doi.org/10.1002/pbc.22384. PMID: 19998467.

9. Hsu HH, Huang CC. Acute spontaneous tumor lysis in anaplastic large T-cell lymphoma presenting with hyperuricemic acute renal failure. Int J Hematol. 2004; 79: 48-51.

10. Sile S, Wall BM. Acute renal failure secondary to spontaneous acute tumor lysis syndrome in myelofibrosis. Am J Kidney Dis. 2001; 38(4): E21.

11. Rampello E, Fricia T, Malaguarnera M. The management of tumor lysis syndrome. Nat Clin Pract Oncol. 2006; 3(8): 438-47. http://doi.org/10.1038/ ncponc0581.

12. Larson RA. Tumor lysis syndrome: prevention and treatment. UpToDate. 2019; 1-22.

13. Trifilio S, Gordon L, Singhal $S$ et al. Reduced-dose rasburicase (recombinant xanthine oxidase) in adult cancer patients with hyperuricemia. Bone Marrow Transplant. 2006; 37(11): 997-1001.

14. Wang $L Y$, Shin $L Y$, Chang $\mathrm{H}$ et al. Recombinant urate oxidase (rasburicase) for the prevention and treatment of tumor lysis syndrome in patients with hematologic malignancies. Acta Haematol. 2006; 115(1-2): 35-8.

Authors' contributions: All authors contributed equally to the article.

Conflict of interests:

Authors declare no conflict of interest.

Financial support:

None.

Ethics:

The authors had full access to the data and take full responsibility for its integrity.

All authors have read and agreed with the content of the manuscript as written. 